\title{
SEOM clinical guidelines for the treatment of follicular non-Hodgkin's lymphoma
}

\author{
M. Provencio Pulla ${ }^{1}$ J. Alfaro Lizaso ${ }^{2}$ L. de la Cruz Merino ${ }^{3} \cdot$ J. Gumá i Padró $^{4}$. \\ C. Quero Blanco ${ }^{5}$ J. Gómez Codina $^{6} \cdot$ M. Llanos Muñoz ${ }^{7} \cdot$ N. Martinez Banaclocha ${ }^{8}$. \\ D. Rodriguez Abreu' - A. Rueda Domínguez ${ }^{10}$
}

Received: 8 October 2015/ Accepted: 9 October 2015/Published online: 19 November 2015

(c) The Author(s) 2015. This article is published with open access at Springerlink.com

\begin{abstract}
Follicular non-Hodgkin's lymphoma (FL) is a nodal B lymphoid malignancy that originates from the germinal center of a lymph node. FL is the second most frequent lymphoma subtype. The course of the disease is usually characterised by a typically indolent clinical course, with a median survival rate of 8-10 years, although most patients relapse after treatment. Diagnosis should
\end{abstract}

M. Provencio Pulla

mprovenciop@gmail.com;

mariano.provencio@salud.madrid.org

1 Servicio de Oncología Médica, Hospital Universitario Puerta de Hierro Majadahonda, Madrid, Spain

2 Servicio de Oncología Médica, Instituto Oncológico de Guipúzcoa, San Sebastián, Spain

3 Servicio de Oncología Médica, Complejo Hospitalario Regional Virgen Macarena, Sevilla, Spain

4 Servicio de Oncología Médica, Hospital Universitari de Sant Joan de Reus, Reus, Spain

5 Servicio de Oncología Médica, Complejo Hospitalario Regional y Virgen de la Victoria, Málaga, Spain

6 Servicio de Oncología Médica, Hospital Universitari i Politècnic la Fe, Valencia, Spain

7 Servicio de Oncología Médica, Hospital Universitario de Canarias (H.U.C), San Cristóbal de La Laguna, Tenerife, Spain

8 Servicio de Oncología Médica, Hospital General Universitario de Elche y Vega Baja, Elche, Spain

9 Servicio de Oncología Médica, Hospital Universitario Insular de Gran Canaria, Las Palmas de Gran Canarias, Spain

10 Área de Oncología y Hematología, Hospital Costa del Sol, Marbella, Spain always be based on a surgical specimen like an excisional node lymph biopsy. The first-line treatment of FL will depend of extension disease, tumour burden, patient symptoms, performance status and also patient decision. The addition of rituximab to conventional chemotherapy has improved ORR, PFS and OS. As first line in patients that need treatment, a combination of chemotherapy with rituximab induction followed by 2 years of rituximab maintenance is the best option. High-dose chemotherapy with autologous stem-cell transplantation in first line has not shown improvement and is not recommended as firstline therapy. Before any treatment decision in relapsed patients, a repeat biopsy is mandatory to rule out a transformation into large cell aggressive lymphoma. Standard treatment is controversial, depends on the efficacy of prior treatment, duration of the time-to-relapse, patient's age and histological findings at relapse.

Keywords Oncohematology malignancies - Follicular non-Hodgkin's lymphoma · Non-Hodgkin lymphoma therapy

\section{Methodology}

To identify the main topics published in medical literature, a search in "PubMed" and "is knowledge"(that includes both full papers and abstracts) has been performed. Key words used were "Non- Hodgkin Lymphoma", "Follicular Lymphoma staging" "Follicular Lymphoma treatment", and "Follicular Lymphoma new therapies".

Main recent reviews on the topics: ESMO clinical guides, NCCN guides, Annual Clinical Updates in Hematology Malignances of the American Journal of Hematology, have been consulted. 


\section{Introduction}

Follicular non-Hodgkin's lymphoma (FL) is a nodal B lymphoid malignancy that originates from the germinal centre of a lymph node [1]. FL is the second most frequent lymphoma subtype, and in recent decades, the incidence has risen to 5-7 cases per 100,000 people and represents approximately $20 \%$ of all lymphomas.

Age-adjusted rate according to European Registry HAEMACARE is 2.18 (IC $95 \%$ 2.12-2.24) per 100.000 people [2]. In a Spanish study conducted between 1999 and 2009, 3651 lymphoid malignancies were registered, $18 \%$ of which were FL, and were the second most common subtype [3].

Most patients with FL suffer from the disease in their sixties or seventies, and it rarely occurs in younger people. The course of the disease is usually characterised by a typically indolent clinical course, with a median survival rate of $8-10$ years, although most patients relapse after treatment [4].

A spontaneous regression of the disease has been reported in nearly $25 \%$ of patients. In many cases, the terminal phase of the disease is associated with transformation to aggressive lymphoma, with an incidence ranging from 16 to $60 \%$.

\section{Diagnosis}

Diagnosis should always be based on a surgical specimen like an excisional node lymph biopsy. Core biopsy should only be performed in patients without accessible lymph nodes (e.g. retroperitoneal node). Fine needle aspirations are inappropriate for a reliable diagnosis.

FL is characterised by a follicular growth pattern, usually composed of a mixture of centrocytes and centroblasts. Grading is performed according to the number of centroblasts observed in a high-power field, and includes grades $1-2$ ( $\leq 15$ blasts), 3 ( $>15$ blasts), 3a (centrocytes still present) and $3 \mathrm{~b}$ with sheets of centroblasts. FL grade $3 \mathrm{~b}$ is considered to be an aggressive lymphoma.

FL has a characteristic immunophenotype, which includes CD19, CD20, CD22, CD79a+, surface immunoglobulins (sIg: IgM, IgD and IgG), Bcl-2+, CD10 \pm , CD5- and CD43-. The chromosome translocation $\mathrm{t}(14: 18)$ (q32;q21), which juxtaposes the bcl-2 gene with the immunoglobin heavy-chain locus, deregulates the expression of the BCL-2 gene in $90 \%$ of FL grade 1 and 2 [5].

In grade $3 \mathrm{FL}$ lacking $\mathrm{t}(14 ; 18)$ it is recommended to perform the study for BCL-6, as it can also be useful in pediatric FL. Gene expression profiles are being studied both in lymphoma cells and in their microenvironment, but they are currently not in use in daily clinical practice [6].

There are three rare clinicopathological variants:

- Bowel primary FL more common in the second portion of the duodenum, in the form of multiple small asymptomatic polyps. Most patients have a localised disease (stage IE or IIE).

- Cutaneous primary FL solitary lesion or few localised lesions (only $15 \%$ presents generalised lesions) in head and trunk (typically, in the back).

- Pediatric FL nodal and extranodal involvement (Waldeyer ring and testicles). It owns unique clinicopathological features: very large follicles, blastoid cytologic features, high proliferation rate and lack of expression of BCL-2 and $\mathrm{t}(14 ; 18)$ (q32; q21). It comes in early stages and is generally associated with a good prognosis.

\section{Staging}

The diagnostic work-up of FL is similar to other lymphomas. Initial work-up should include clinical history and physical examinations, paying special attention to the lymph nodes, liver and spleen; a complete blood count; routine blood chemistry, including liver and renal function, lactate dehydrogenase (LDH) levels, uric acid levels, immunoglobulin levels and $\beta 2$ microglobulin levels; as well as screening tests for HIV, hepatitis B and hepatitis C. A computed tomography (CT) of neck, thorax, abdomen and pelvis and bone marrow biopsy have to be performed.

The recent Consensus of the International Conference Lymphoma Working Group recommends performing PETTC [7]. In case of histology transformation suspicion, PET can identify the optimal site for biopsy. It is also useful in the early stages that will be treated with radiation therapy to confirm localised disease [IV, C].

FL staging is typically given according to the Ann Arbor system [8]. (Table 1). Lugano Classification no longer recommends the addition of $\mathrm{B}$ symptoms or the use of $\mathrm{X}$ for bulky disease in FL [9].

Table 1 Cotswolds/Ann Arbor staging system

Stage I Single lymph node group or extranodal (IE)

Stage II Multiple lymph node groups on the same side of the diaphragm or extranodal and one or more lymph node (IIE) on the same side of the diaphragm

Stage III Multiple lymph node groups on both side of the diaphragm

Stage IV Presence of diffuse or disseminated involvement of one or more extralymphatic organs 
Table 2 Follicular lymphoma international prognostic index (FLIPI)

\begin{tabular}{lllll}
\hline FLIPI score & No. of risk factors & Patients $(\%)$ & 5 years survival $(\%)$ & 10 years survival $(\%)$ \\
\hline Good & $0-1$ & 36 & 90.6 & 70.7 \\
Intermediate & 2 & 37 & 77.6 & 50.9 \\
High & $3-5$ & 27 & 52.5 & 35.5 \\
\hline
\end{tabular}

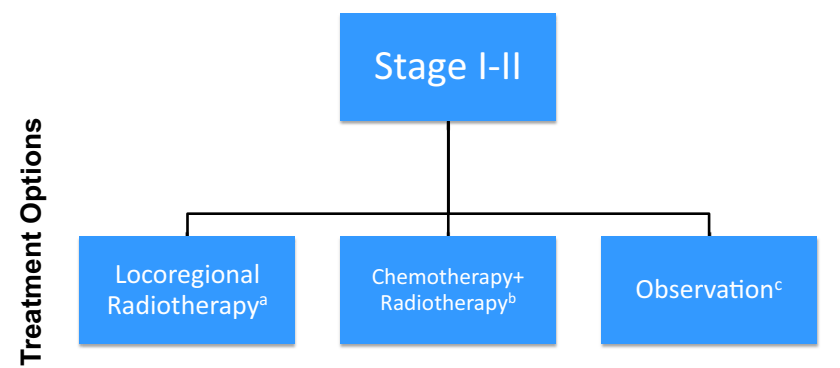

a30-40 Gy is the preferred treatment option.

bImprove failure-free survival but not overall survival.

cIn case where toxicity of radiotherapy outweighs potential clinical benefit.

Fig. 1 Treatment algorithm of follicular lymphomas (Grade 1-2) localised disease

Most patients present advanced stage lymphoma, directing the need for more information for prognostic purposes. The Follicular Lymphoma-specific International Prognostic Index (FLIPI) has established 5 risk factors: more than four of these factors involve node sites, elevated LDH levels, age >60 years, advanced III-IV stage and haemoglobin levels $<12 \mathrm{~g} / \mathrm{dl}$. FLIPI stratifies patients into three different risk categories: low (0-1), intermediate (2) and high (3-5) risk for overall survival, with different rates of survival [10] [I, A] (Table 2).

FLIPI-2 [11] has been developed in a prospective study of patients treated with rituximab. Factors that include FLIPI-2 are the following: age $>60$ years, bone marrow infiltration, haemoglobin $<12 \mathrm{~g} / \mathrm{dl}$, high $\beta 2$ microglobulin and lymph node diameter $>6 \mathrm{~cm}$.

\section{Treatment}

\section{First-line treatment}

The first-line treatment of FL will depend on the extension of the disease, tumour burden, patient symptoms, performance status (PS) and also the patient's decision. (Fig. 1).

\section{Stage I-II disease}

Only 15-25 \% of patients are diagnosed with non-bulky Ann Arbor stage I/II. In asymptomatic patients, initial observation is a valid option [IV, B] [12, 13]. In symptomatic patients, Involved Field Radiation (IFR)
(24-36 Gy) is the most recommended treatment worldwide, achieving complete responses (CR) up to $97 \%$ of cases and long-term disease control with most recurrences outside radiation field [II, B] [14, 15]. However, there are no randomised studies against other strategies and patients involved in the studies with radiation therapy are heterogeneous with different doses and schedules used over the time. The addition of chemotherapy has not demonstrated any further benefits and anti-CD20 therapy has not been adequately studied in limited-stage FL [IV, C] [16]. Observation or Rituximab (R) monotherapy may be an option to avoid radiotherapy side effects with no deleterious impact in overall survival (OS) [IV, B] [17]. In cases of high tumour burden, patients can be treated with chemoimmunotherapy like in the advanced setting before radiation therapy [IV, B] [18].

\section{Stage III-IV disease}

To date, advanced stage FL was considered incurable with conventional treatment strategies. But recently, the plateau observed in the survival curves at 10 years in different series indicates the existence of long-term survivors in the $\mathrm{R}$ era. Therefore, the goal of treatment is to achieve the best response and prolong progression-free survival (PFS) and, if possible, prolong OS (Fig. 2).

The decision to treat and the selection of treatment should be individualised based on the patient symptoms, tumour burden, hematopoietic impairment, histological transformation and comorbid conditions. In general, GELF (Groupe pour l'Etude de Lymphome Folliculaire) criteria are used to initiate treatment [19] (Table 3).

Both before the era of $\mathrm{R}$ and at present, in asymptomatic patients with low tumour burden, the strategy of "wait and see" has had no negative impact on patient survival as demonstrated in different randomised trials [16]. So, taking into account that median age of advanced FL patients is between 60 and 65 years and spontaneous regressions are described in about 10-20\% of cases, asymptomatic patients can be managed with a wait and see strategy [I, A]

Patients with indication for treatment should be treated with systemic therapy and, if not contraindicated, associated with $\mathrm{R}$. Inclusion in clinical trials with new agents should alwaysobservation is a valid opti be considered as the first line of treatment. 


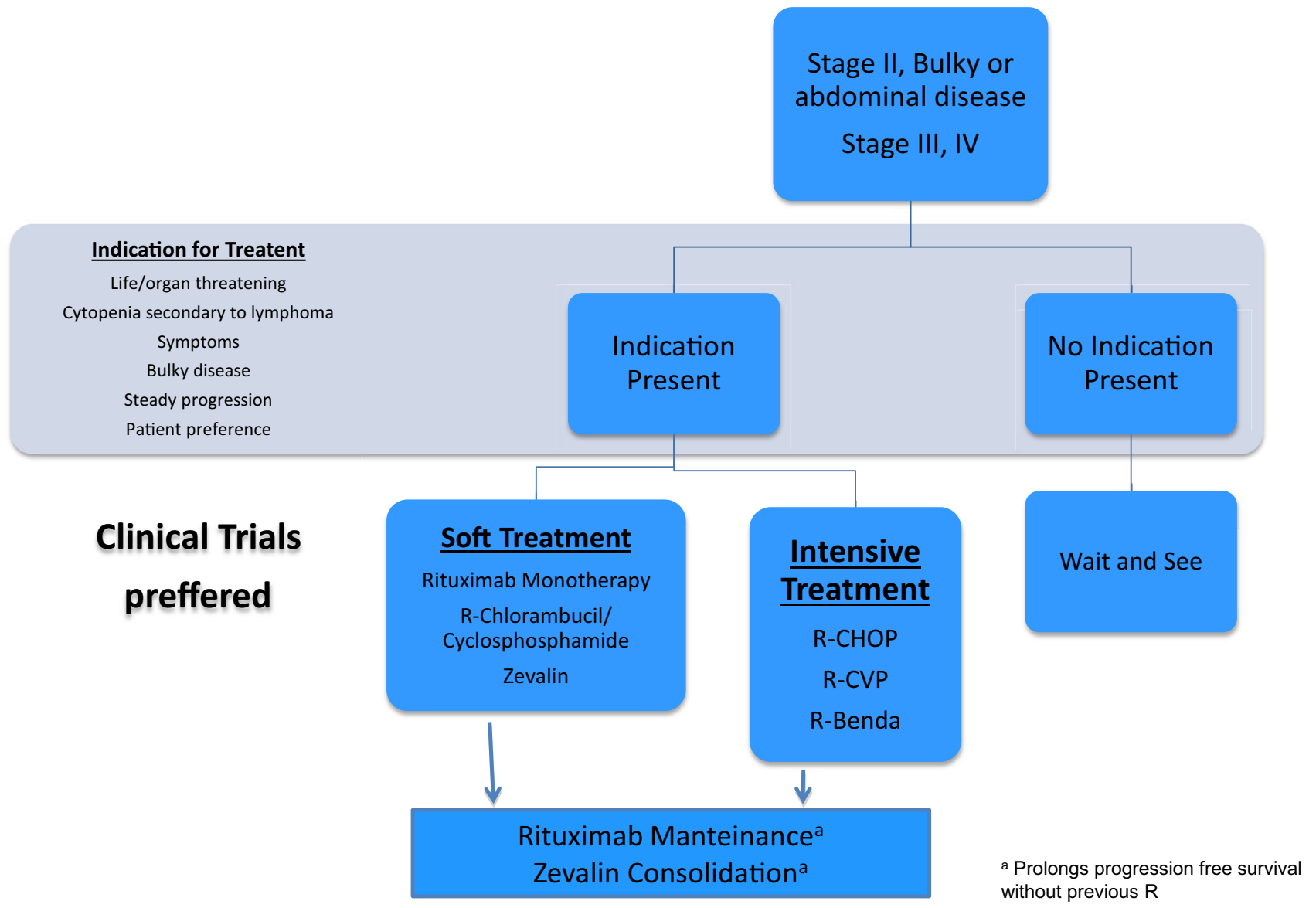

Fig. 2 Treatment algorithm for advance disease

\section{Table 3 GELF Criteria}

- Any nodal or extranodal tumour mass (except spleen) with a diameter $>7 \mathrm{~cm}$

- Involvement of more than three nodal sites with a diameter $>3 \mathrm{~cm}$

- Presence of systemic symptoms

- Substantial splenic enlargement (symptomatic or $>16 \mathrm{~cm}$ on computed tomography)

- Pleural or peritoneal effusions

- Risk of local compression (epidural, intestinal, ureteral and orbital among others)

- Circulating lymphoma cells $(>5 \times 109 / 1$ malignant cells)

- Marrow compromise (haemoglobin $<10 \mathrm{~g} / \mathrm{dl}$, granulocytes count $<1.5 \times 109 / 1$ or platelet count $<100 \times 109 / 1$

- ECOG PS $\geq 2$

- Elevated serum LDH or B-2 microglobulin

The addition of $\mathrm{R}$ to conventional chemotherapy has improved ORR, PFS and OS in different trials and one metaanalysis, including first line and salvage therapy settings [20] [I, A]. R-CHOP schedule is the most widely used worldwide but other schemes also can be used according to patient
Table 4 First line: Different schemes of chemoimmunotherapy

\begin{tabular}{llll}
\hline Combination & ORR $(\%)$ & PFS 3y $(\%)$ & Neutropenia 3, $4(\%)$ \\
\hline R-CVP & 88,91 & 46, ND & 28,56 \\
R-CHOP & 93,91 & $62, \mathrm{ND}$ & 50,87 \\
R-FM & 91 & $59, \mathrm{ND}$ & 64 \\
R-B & 97 & ND & 39 \\
\hline
\end{tabular}

$R$-CVP rituximab, cyclophosphamide, vincristine, prednisolone; $R$ $C H O P$ rituximab, doxorubicin, cyclophosphamide, vincristine, prednisolone; $R-F M$ rituximab, fludarabine, mitoxantrone; $R-B$ rituximab, bendamustine; $N D$ no data; ORR overall response rate; $P F S$ progression-free survival

characteristics without significant differences in overall survival $[21,22]$ [I, B]. The use of fludarabine has been declining because of drug toxicity profile that may hinder the further management of the patient. The combination of R-Bendamustine as induction therapy has demonstrated increased PFS and OS compared with R-CHOP in first-line treatment of advanced LF in a phase III study. However, this study was performed without maintenance $\mathrm{R}$ so it is unclear whether induction with R- Bendamustine is comparable to 
Fig. 3 Treatment algorithm for relapsed or refractory follicular lymphoma

\section{Second-line therapy}

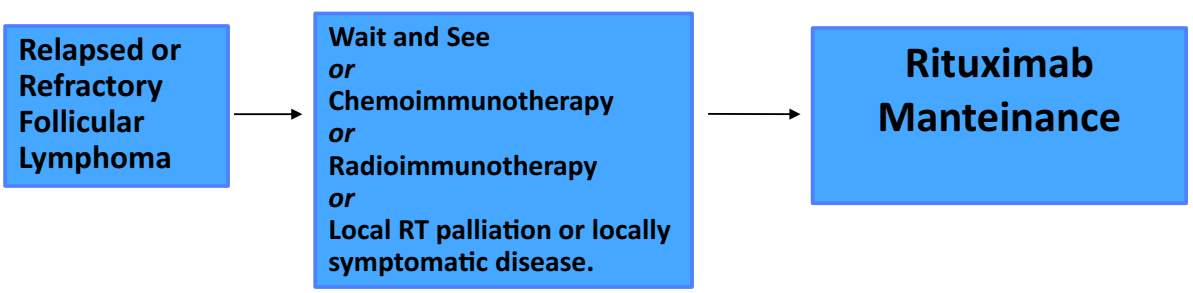

treatment with R-CHOP induction plus 2 years of maintenance with $\mathrm{R}$ [23] [I, B] (Table 4).

Regarding maintenance therapy, the results from the Primary Rituximab and Maintenance (PRIMA) phase III trial have demonstrated that maintenance therapy results in longer PFS, although there is no impact in overall survival (OS) [24] [I, B]. A meta-analysis suggested the use of interferon maintenance therapy as part of first-line treatment [II, B]; however, due to poor toxicity profile front of $\mathrm{R}$ has made the latter preferred in this setting [III, B].

Radioimmunotherapy consolidation prolongs PFS after chemotherapy, although its benefit following $\mathrm{R}$ combinations has not been established as a first-line treatment [25]. It can be an option for patients who are unable to tolerate standard chemotherapy (elderly or unfit patients) or in high-risk patients achieving a PR or CR after induction therapy [II, B].

In conclusion, as first line in patients that need treatment, a combination of chemotherapy with rituximab induction followed by 2 years of rituximab maintenance is the best option [I, B].

For low-risk or unfit patients, the use of single agents (chlorambucil and cyclophosphamide), R monotherapy, radioimmunotherapy, or a combination of $\mathrm{R}$-chlorambucil and R-cyclophosphamide is a good choice to consider [III, B].

\section{Radiation therapy is only used for palliation in locally symptomatic disease [II, B]}

High-dose chemotherapy with autologous stem-cell transplantation in first line has not shown improvement and is not recommended as first-line therapy, this strategy should be used only in clinical trials [26] [I, D].

\section{Second-line treatment}

Before any treatment decision in relapsed patients, a repeat biopsy is mandatory to rule out a transformation into large cell aggressive lymphoma (Fig. 3).

Clinical trial participation is always recommended in relapsed or refractory follicular lymphoma. Standard treatment is controversial, and depends on the efficacy of prior treatment, duration of the time-to-relapse, patient's age and histological findings at relapse.

Retreating with the same chemotherapy that was effective is reasonable especially after a long remission. Nevertheless, a non-cross-resistant scheme should be preferred (bendamustine after CHOP) [I,B]. The addition of rituximab should be considered if previously it was able to achieve a durable remission and then to continue with rituximab maintenance every 3 months which has demonstrated to significantly improve OS, compared with observation or treatment with the drug at the time of disease progression, according to a new meta-analysis [I, A].

Radioimmunotherapy has been demonstrated to be an effective second-line treatment even in patients who have FL recurrence after rituximab exposure [27] [I, B].

High-dose chemoradiotherapy with autologous hematopoietic cell transplantation is effective in relapse FL but its role has to be redefined in the rituximab era. Allogeneic stem-cell transplantation can provide durable longterm molecular remission but with high treatment-related mortality, reserving this modality for young and very motivated patients, with suitable donors [28].

Different promising new agents are currently being explored for the treatment of patients with relapsed or refractory FL, new antibodies (targeting CD20, CD 22 and CD23, such as Obinutuzumab, epratuzumab and lumiliximab); new drugs targeting oncogenic pathways (PI3K, such as idelalisib or Brutońs tyrosine kinase inhibitors such as ibrutinib); immunotherapy (anti-PD1 as Pidilizumab).

\section{Compliance with ethical standards}

Conflict of interest The authors declare that they have no conflict of interest.

Open Access This article is distributed under the terms of the Creative Commons Attribution 4.0 International License (http:// creativecommons.org/licenses/by/4.0/), which permits unrestricted use, distribution, and reproduction in any medium, provided you give appropriate credit to the original author(s) and the source, provide a link to the Creative Commons license, and indicate if changes were made. 


\section{References}

1. Campo E, Swerdlow SH, Harris NL, Pileri S, Stein H, Jaffe ES. The 2008 WHO classification of lymphoid neoplasms and beyond: evolving concepts and practical applications. Blood. 2011;117:5019-32.

2. Sant M, Allemani C, Tereanu C, De Angelis R, Capocaccia R, Visser O, et al Incidence of hematologic malignancies in Europe by morphologic subtype: results of the HAEMACARE Project. Blood. 2010;116:3724-34.

3. Provencio M, Sabin P, Gómez-Codina J, Rueda A, Llanos M, Gumá J, et al. Are there any significant variations in the clinical or histological presentation of lymphoid pathologies over the course of time in Spain? Clin Transl Oncol. 2012;14:386-90.

4. Marcos-Gragera R, Allemani C, Tereanu C, De Angelis R, Capocaccia R, Maynadie M, et al. Survival of European patients diagnosed with lymphoid neoplasms in 2000-2002: results of the HAEMACARE project. Haematologica. 2011;96(5):720-8.

5. Montes S, Mollejo M, Fraga M, García JF, Villar JL, Martínez A, et al. Recomendaciones para el estudio histopatológico, inmunohistoquímico, citogenético y molecular e informe diagnóstico de los procesos neoplásicos linfoides. Consenso SEAP-SEHH-GOTEL. 1a edición. Mayo 2015.

6. Dreyling M, Ghielmini M, Marcus R, Salles G, Vitolo U, Ladetto M. Newly diagnosed and relapsed follicular lymphoma: ESMO Clinical Practice Guidelines for diagnosis, treatment and follow-up. Ann Oncol. 2014;25(Supplement 3):iii76-82.

7. Barrington SF, Mikhaeel NG, Kostakoglu L, Meignan M, Hutchings M, Stefan $\mathrm{P}$, et al. Role of imaging in the staging and response assessment of lymphoma: consensus of the international conference on malignant lymphomas imaging working group. J Clin Oncol. 2014;32:3048-58.

8. Lister TA, Crowder D, Sutcliffe SB, Glatstein E, Canellos GP, Young RC, et al. Report of a committee convent to discuss the evaluation and staging of patients with Hodgkin's lymphoma: Cotswolds meeting. J Clin Oncol. 1989;7:1630-6.

9. Cheson BD, Fisher RI, Barrington SF, Cavalli F, Schwartz LH, Zucca E, et al. Recommendations for initial evaluation staging, and response assessment of Hodgkin and non-Hodgkin lymphoma: the Lugano classification. J Clin Oncol. 2014;32(27):3059-68.

10. Solal-Celigny P, Roy P, Dreyling M, White J, Armitage JO, Arranz-Saez R, et al. The follicular lymphoma prognostic index (FLIPI). Bood. 2004;104:1258-65.

11. Federico M, Bellei M, Marcheselli L, Luminari S, Lopez-Guillermo A, Vitolo $\mathrm{U}$, et al. Follicular lymphoma international prognostic index 2 : a new prognostic index for folicular lymphoma developed by the international folicular lymphoma prognostic factor project. J Clin Oncol. 2009;27:4555-62.

12. Advani R, Rosenberg SA, Horning J. Stage I and II follicular non-Hodgkin's lymphoma: long-term follow-up of no initial therapy. J Clin Oncol. 2004;22:1454-9.

13. Hoskin PJ, Kirkwood AA, Popova B, Smith P, Robinson M, Gallop-Evans E, et al. 4 Gy versus 24 Gy radiotherapy for patients with indolent lymphoma (FORT): a randomised phase 3 non-inferiority trial. Lancet Oncol. 2014;15(4):457-63

14. Barzenje DA, Cvancarova Småstuen M, Liestøl K, Fosså A, Delabie J, Kolstad A, et al. Ra diotherapy compared to other strategies in the treatment of stage I/II follicular lymphoma: a study of 404 patients with a median follow-up of 15 Years. PLoS One. 2015. doi:10.1371/journal.pone.0131158:1-16.

15. Guadagnolo BA, Li S, Neuberg D, Nq A, Hua L, Silver B, et al. Long-term outcome and mortality trends in early-stage, grade 1-2 follicular lymphoma treated with radiation therapy. Int J Radiat Oncol Biol Phys. 2006;64:928-34.
16. Solal-Céligny P, Bellei M, Marcheselli L, Pesce EA, Pileri S, McLaughlin P, et al. Watchful waiting in low-tumor burden follicular lymphoma in the rituximab era: results of an F2-study database. J Clin Oncol. 2012;30(31):3848-53.

17. Friedberg JW, Byrtek M, Link BK, Flowers C, Taylor M, Hainsworth J, et al Effectiveness of first-line management strategies for stage I follicular lymphoma: analysis of the National LymphoCare Study. J Clin Oncol. 2012;30:3368-75

18. Salles GA, Seymour JF, Feugier P, Offner F, LopezGuillermo A, Belada D, et al. Updated 6 year follow-up of the PRIMA study confirms the benefit of 2-year rituximab maintenance in follicular lymphoma patients responding to frontline immunochemotherapy. Blood. 2013;122(21):509.

19. Ardeshna KM, Smith P, Norton A, Hancock BW, Hoskin PJ, MacLennan KA, et al. Long-term effect of a watch and wait policy versus immediate systemic treatment for asymptomatic advanced-stage non-Hodgkin lymphoma: a randomised controlled trial. Lancet. 2003;362(9383):516-22.

20. Federico M, Luminari S, Dondi A, Tucci A, Vitolo U, Rigacci L, et al. R-CVP versus R-CHOP versus R-FM for the initial treatment of patients with advancedstage follicular lymphoma: results of the FOLL05 trial conducted by the Fondazione Italiana Linfomi. J Clin Oncol. 2013;31:1506-13.

21. Flinn I, van der Jagt R, Kahl RS, Wood P, Hawkins TE, Macdonald D, et al. Randomized trial of bendamustine-rituximab or R-CHOP/R-CVP in first-line treatment of indolent NHL or MCL: the BRIGHT study. Blood. 2014;123(19):2944-52.

22. Rummel M, Niederle N, Maschmeyer G, Banat GA, won Grünhagen U, Losem $\mathrm{C}$, et al. Bendamustine plus rituximab versus CHOP plus rituximab as first-line treatment for patients with indolent and mantle- cell lymphoma: an open-label, multicentre, randomised, phase 3 non-inferiority trial. Lancet. 2013;381:1203-10

23. Salles G, Seymour JF, Offner F, López-Guillermo A. Rituximab maintenance for 2 years in patients with high tumour burden follicular lymphoma responding to rituximab plus chemotherapy (PRIMA): a phase 3, randomised controlled trial. Lancet. 2011;377(9759):42-51.

24. Vidal L, Gafter-Gvili A, Leibovici L, Dreyling M, Ghielmini M, Hsu Schmitz $\mathrm{SF}$, et al. Rituximab maintenance for the treatment of patients with follicular lymphoma: systematic review and meta-analysis of randomized trials. J Natl Cancer Inst. 2009;101:248-55.

25. Provencio M, Cruz Mora MÁ, Gómez-Codina J, Quero Blanco C, Llanos M, García-Arroyo FR, et al. Gotel (Spanish Lymphoma Oncology Group). Consolidation treatment with Yttrium-90 ibritumomab tiuxetan after new induction regimen in patients with intermediate- and high-risk follicular lymphoma according to the follicular lymphoma international prognostic index: a multicenter, prospective phase II trial of the Spanish Lymphoma Oncology Group. Leu Lymphoma. 2014;55(1):51-5.

26. Montoto S, Corradini P, Dreyling M, Ghielmini M, Kimby E, López-Guillermo A, et al. Indications for hematopoietic stem cell transplantation in patients with follicular lymphoma: a consensus project of the EBMT-Lymphoma Working Party. Haematologica. 2013;98(7):1014-21.

27. Kaminski MS, Estes J, Zasadny KR, Francis IR, Ross CW, Tuck M, et al. Radioimmunotherapy with iodine (131)I tositumomab for relapsed or refractory B-cell non-Hodgkin lymphoma: updated results and long-term follow-up of the University of Michigan experience. Blood. 2000;96(4):1259-66.

28. Schouten HC, Qian W, Kvaloy S, Porcellini A, Hagberg H, Johnsen HE, et al High-dose therapy improves progression-free survival and survival in relapsed follicular non-Hodgkin's lymphoma: results from the randomized European CUP trial. J Clin Oncol. 2003;21(21):3918-27. 